\section{Defibrillator in der Praxis obligatorisch?}

\author{
Für den Autor des folgenden Leserbriefs erwächst aus der aktuellen \\ Leitlinie zur Anaphylaxie (Allergo J 2007; 16: 420-34) eine haftungs- \\ rechtliche Problematik.
}

$\mathrm{Mi}$ it großem Interesse habe ich die Veröffentlichung der Leitlinien der allergologischen Fachgesellschaften zur „Akuttherapie anaphylaktischer Reaktionen", publiziert im Allergo Journal vom September 2007 von einem Autorenteam um Prof. Dr. Dr. Johannes Ring, zur Kenntnis genommen. Die Leitlinie ist für mich sehr relevant, da ich selbst Notfallseminare zur Akuttherapie im Rahmen allergologischer Tätigkeit durchführe, unter anderem auch bei den letzten beiden DDG-Tagungen in Dresden.

Eine Frage zur Notfallausstattung einer allergologischen Praxis bleibt für mich nach der Lektüre allerdings ungeklärt: Konkret wird in Tabelle 3 die erforderliche Notfallausrüstung zur Behand-

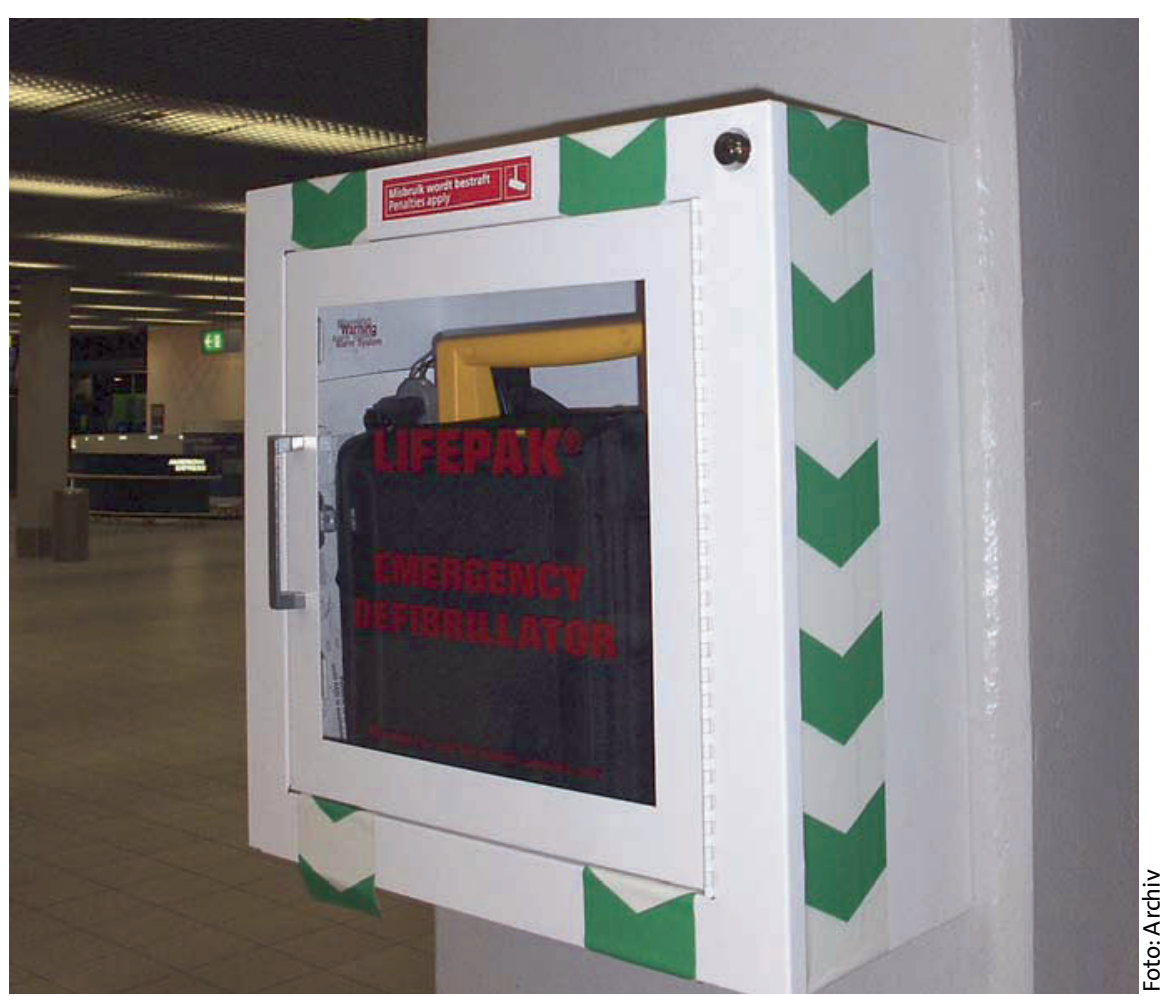

An Flughäfen oder in U-Bahn-Stationen inzwischen ein alltäglicher Anblick, gehört der externe Defibrillator noch nicht in jeder Arztpraxis zum Standard. allergologische Diagnostik noch eine Hyposensibilisierung durchführen darf?

Für eine Klärung dieser Fragen wäre ich dankbar, und mich würde zu diesem Punkt auch die Meinung anderer Leser des Allergo Journals interessieren.

\section{Dr. Elmar Ehring \\ Hautarztpraxis \\ Marktallee 26 \\ 48165 Münster-Hiltrup}

lung anaphylaktischer Reaktionen aufgeauch ein ,automatischer externer Defibrillator". Mit der Aufnahme des Defibrillators in die Notfallausrüstung zur Behandlung anaphylaktischer Reaktionen wird eine Diskussion neu entfacht, die vor einiger Zeit schon in Bezug auf die operative Tätigkeit in der Praxis erfolgte. Meine Frage ist nun: Wie bindend sind Leitlinien in diesem Fall? Wie werden Gutachter und schließlich auch Richter das Fehlen eines Defibrillators beim Eintritt eines wirklichen schweren Zwischenfalls interpretieren? Bedeutet die Vorgabe, die die neue Leitlinie hier macht, nicht in letzter Konsequenz, dass man ohne Vorhaltung eines Defibrillators weder eine

\section{Stellungnahme}

$D_{d}$ ie Debatte um Defibrillatoren und deren Vorhaltung in der allergologischen Praxis ist nicht neu, hat aber durch die Entwicklung von automatisierten externen Defibrillatoren (AED) eine neue Wendung genommen. Leitlinien sollten vor allem als Handlungsanleitung und Orientierungshilfe verstanden werden. Die Nennung des AED zusätzlich zum Notfallkoffer ist aus notfallmedizinischer Sicht dringend empfehlenswert, derzeit aber (noch) nicht vorgeschrieben. Auf jeden Arzt wird aber sicherlich zunehmend Erklärungsbedarf zukommen, wenn Sicherheitsbeamte in U-Bahnen, Kabinenpersonal in Flugzeugen oder Kaufhausdetektive die Defibrillation mit AEDs (problemlos) durchführen, diese aber im Rahmen originärer ärztlicher Behandlungsmaßnahmen in der eigenen Praxis unmöglich ist.

Defibrillation ist zweifelsfrei der Goldstandard der Reanimation (bei beobachtetem Herzstillstand) des Erwachsenen, wie gerade in einer Arztpraxis zu erhoffen. Die öffentliche Verbreitung im Sinne von "public access" trägt dieser Tatsache Rechnung. Die Datenlage für diese Priorität der schnellstmöglichen Defibrillation in den ersten Minuten nach Kreislaufstillstand basiert auf folgenden Erkenntnissen: Die mit Abstand häufigste Form des funktionellen Herzstillstandes (60-70\%) beim Erwachsenen ist Kammerflimmern. Jede Zeitverzögerung bis zur Defibrillation senkt entscheidend die Reanimations- und Überlebenswahrscheinlichkeit des Patienten. Jede Minute Verzögerung bedeutet einen Anstieg der Mortalität. 